\title{
The relationship between ambulatory arterial stiffness index and left ventricular diastolic dysfunction in HFpEF: a prospective observational study
}

\section{Zhiqiang Liu ( $\sim$ zhiqiangfda@qq.com )}

First Affiliated Hospital of Chongqing Medical University

Hanwen Zhang

First Affiliated Hospital of Chongqing Medical University

Weiwei Hu

First Affiliated Hospital of Chongqing Medical University

\section{Yu Wang}

First Affiliated Hospital of Chongqing Medical University

Jie Liu

First Affiliated Hospital of Chongqing Medical University

\section{Linna You}

First Affiliated Hospital of Chongqing Medical University

Qian Dong

First Affiliated Hospital of Chongqing Medical University

\section{Guanglei Chang}

First Affiliated Hospital of Chongqing Medical University

\section{Xiaocheng Cheng}

First Affiliated Hospital of Chongqing Medical University

\section{Dongying Zhang}

First Affiliated Hospital of Chongqing Medical University

\section{Research Article}

Keywords: ambulatory arterial stiffness index, diastolic function, heart failure with preserved ejection fraction

Posted Date: February 21st, 2022

DOI: https://doi.org/10.21203/rs.3.rs-1319474/v1 
License: (c) (i) This work is licensed under a Creative Commons Attribution 4.0 International License. Read Full License 


\section{Abstract}

Background The relationship between ambulatory arterial stiffness index (AASI) and left ventricular diastolic dysfunction in patients with heart failure with preserved ejection fraction (HFpEF) is unknown. We aimed to investigate whether increased AASI was related to left ventricular diastolic dysfunction in patients with HFpEF.

Methods We prospective enrolled consecutive patients with HFpEF in Chongqing, China. Twenty-four-hour ambulatory blood pressure monitoring (24h-ABPM) and echocardiography were performed in each patient. AASI was obtained through individual 24h-ABPM. The relationship between AASI and left ventricular diastolic function was analyzed.

Results A total of 91 patients with HFpEF were included. The mean age was $68.7 \pm 13.9$ years and 54 $(59 \%)$ were women. The patients were divided into two groups according to the upper normal border of AASI (0.55). AASI > 0.55 group were more likely to be older, to have higher mean systolic blood pressure and worsen left ventricular diastolic function than AASI group $\leq 0.55$. AASI was closely positive related to the diastolic function parameters, including mean $E / e^{\prime}(r=0.290, P=0.005)$, septal $E / e^{\prime}(r=0.237, P=$ $0.024)$, lateral $E / e^{\prime}(r=0.295, P=0.004)$ and $E(r=0.262, P=0.012)$. After adjusting for conventional risk factors, AASI was still an independent risk factors of left ventricular diastolic dysfunction in patients with HFpEF (odds ratio: $3.261,95 \% \mathrm{Cl}: 1.099-9.673, \mathrm{P}=0.033$ ).

Conclusion Increased AASI was independent associated with left ventricular diastolic dysfunction in patients with HFpEF.

\section{Introduction}

Ambulatory arterial stiffness index (AASI) is defined as 1 minus the regression slope of diastolic on systolic blood pressure (BP) values obtained from the 24-hour ambulatory blood pressure monitoring (ABPM) recordings ${ }^{1,2}$. It has been proposed as a novel indicator of arterial stiffness and has advantages by the low cost and noninvasive. Substantial reports revealed that increased arterial stiffness was associated with preclinical target organ damage and increased risk of cardiovascular mortality and morbidity in hypertension $3,4,5$.

Heart failure with preserved ejection fraction (HFpEF) is characterized by diastolic dysfunction and cardiac remodeling (fibrosis, inflammation, and hypertrophy), which has become a major cause of hospitalization for the elders ${ }^{6,7,8}$. Hypertension, diabetes and obesity were risk factors for HFpEF and were associated with arterial stiffness raise $e^{9,10}$. It was reported that microvascular dysfunction and chronic low-grade inflammation have been proposed to participate in HFpEF development ${ }^{11}$. Other parameters reflecting arterial stiffness such as cardio-ankle vascular index (CAVI), has been reported associated with the hospitalization of HFpEF patients ${ }^{12}$, but the role of AASI in HFpEF is still unknown. 
The objective of the present study was to investigate the relationship between ambulatory arterial stiffness index (AASI) and left ventricular diastolic dysfunction in patients with HFpEF.

\section{Methods}

\section{Study design and participants}

From November 2020 to February 2021, we conducted a prospective observational study registry with clinicaltrials.gov identifier NCT05059769. This study finally enrolled 91 patients with HFpEF in the First Affiliated Hospital of Chongqing Medical University (Fig. 1). Inclusion criteria included age > 18 years and conform to HFpEF diagnostic criteria (Left ventricular ejection fraction $\geq 50 \%$, typical symptoms and

signs of heart failure, HFA-PEFF score $\geq 5)^{13}$, whereas the exclusion criteria were secondary hypertension, severe valvular heart disease and persistent atrial fibrillation. Informed consent was obtained from the patients, and the study was approved by the institutional ethics board of the First Affiliated Hospital of Chongqing Medical University (approval N0.2020-606). Baseline clinical and demographic information was obtained from all patients. Body mass index (BMI) was calculated as weight $(\mathrm{kg}) / \mathrm{height}\left(\mathrm{m}^{2}\right) .24-$ hour ABPM and echocardiography were carried out in all patients during hospitalization.

\section{4-hour ABPM}

24-hour ABPM was performed using the Mobil-O-Graph NG (Z02505), a non-invasive ambulatory BP monitoring instrument. BP readings were obtained at $15-\mathrm{min}$ intervals during the day and at $30-\mathrm{min}$ intervals during the night. Of the total readings, $\geq 80 \%$ was considered valid. Furthermore, for the records valuable, at least 14 measurements during the daytime period or at least 7 measurements during the night or rest period were required ${ }^{14}$.

\section{Ambulatory arterial stiffness index (AASI)}

AASI is a more physiologically significant marker of arterial stiffness than a simple snapshot of pulse wave velocity or pulse pressure, which is derived from the regression slope of diastolic BP and systolic BP recorded in unedited 24 hours. Based on the 24-hour ABPM records, we calculated the regression slopes of individual patients' diastolic BP and systolic BP. The stiffer the arterial tree, the closer the regression slope and AASI are to 0 and 1 , respectively ${ }^{1,2}$. AASI was obtained as follows:

AASI = 1-slope (diastolic BP/systolic BP)

\section{Echocardiography}


The cardiac diastolic function of HFpEF was assessed by transthoracic echocardiography (Vivid E95, AU11403, GE Vingmed Ultrasound AS). Using the parasternal short-axis two-dimensional view to image the heart and record an M-mode echocardiogram at the level of the papillary muscles. Cardiac function parameters, such as left ventricular ejection fraction (LVEF), diastolic interventricular septum thickness (IVSd), diastolic left ventricular posterior wall thickness (LVPWd), left atrium volume index (LAVI), left ventricle mass index (LVMI), the peak velocity of the filling peak in the early diastolic period (E) , the peak velocity of the filling peak in the late diastolic period $(A)$, the $E / A$ ratio $(E / A)$, the ratio of the early diastolic transmitral filling velocity to the early diastolic septal tissue velocity (Septal E/e') and the ratio of early diastolic transmitral flow velocity to mitral annular velocity at the lateral wall (Lateral E/e') were measured by the same investigator. At the same time, tricuspid annular plane systolic excursion (TAPSE) and plane contraction offset velocity of tricuspid annulus (TAPSE-S) reflecting right ventricular function were measured as well ${ }^{15}$.

\section{Data collection}

Data on epidemiological information, medical history, exposure history, underlying comorbidities, symptoms, signs, laboratory, and radiological characteristics were obtained from electronic medical records. All the data were collected by two investigators independently and double checked by other investigators.

\section{Definition}

Not all patients had severe diastolic dysfunction, so in the logistic regression, we set mean E/e' $=10$ rather than 14 as the critical value. AASI $=0.52$ was the cut-off point of receiver operating characteristic (ROC) curves (Fig 3). It was close to the upper normal border of AASI $=0.55^{1,2}$, so we pre-specified that the patients would be divided into two groups according to the upper normal border: AASI group $\leq 0.55$ group and $A A S I>0.55$ group.

\section{Statistical analysis}

Categorical variables were described as frequency rates and percentages, and continuous measurements as mean (standard deviation: [SD]) if they are normally distributed or median (interquartile range: [IQR)] if they are not. $X^{2}$ test was used to test for differences in categorical variables among the two groups. $T$ test or Mann-Whitney test was used to compare continuous variables according to the normal distribution or not. Spearman correlation analysis was used for assessing the correlates of left ventricular diastolic function. logistic regression analysis was used to test independent factors of left ventricular diastolic dysfunction. To explore whether AASI provides additional value in predicting impaired left ventricular diastolic function in patients with HFpEF, we performed ROC curves and tested for equality of the areas 
under the curves (AUC). All P values were two-tailed, and significance was set at $P<0.05$. Statistical analyses were performed using SPSS software (version 22.0).

\section{Results}

\section{Baseline characteristics}

The study initially enrolled 129 consecutive patients with HFpEF. Patients who had secondary hypertension $(n=8)$, severe valvular heart disease $(n=10)$ and persistent atrial fibrillation $(n=20)$ were excluded (Fig. 1). Finally, a total of $91 \mathrm{HFpEF}$ patients were included. The patients were divided into two groups according to the upper normal border of AASI (0.55). Table 1 showed the overall baseline clinical and demographic data in two groups. The mean age of the patients was $68.73 \pm 13.93$ years. More than half had comorbidities including hypertension $(60,66 \%)$, diabetes $(30,33 \%)$ and coronary artery disease $(41,45 \%)$. Age, ave-systolic BP, LAVI, E, septal E/e', lateral E/e' and mean E/e' in the AASI > 0.55 group were significantly higher than the AASI group $\leq 0.55$. There was no significant difference in the other clinical parameters between the two groups, including comorbidities, height, weight, BMI and LVEF. We also focused on the right ventricular systolic function, in which the TAPSE and TAPSE-S did not differ between the two groups.

\section{Spearman correlations between clinical characteristics and left ventricular diastolic function}

The relationships for AASI and other clinical parameters between left ventricular diastolic function, including $\mathrm{E}$, septal E/e', lateral E/e' and mean E/e' were conducted by spearman correlations analysis. The results were shown in Table 2. We found that AASI was closely positive related to the diastolic function parameters, including mean $E / e^{\prime}\left(r=0.290, P=0.005\right.$; Fig.2a), septal $E / e^{\prime}(r=0.237, P=0.024 ;$ Fig. $2 b)$, lateral $E / e^{\prime}(r=0.295, P=0.004$; Fig.2c) and $E(r=0.262, P=0.012$; Fig.2d). The proportion of patients with $\mathrm{E} / \mathrm{e}^{\prime}>10$ was $35(76 \%)$ in the AASI $>0.55$ group, which was higher than that in the AASI $\leq 0.55$ group $(25,53 \%, P=0.023)$. Age and ave-systolic BP were also related to mean $E / e^{\prime}$, septal $E / e^{\prime}$ and lateral $\mathrm{E} / \mathrm{e}^{\prime}$. BMI was related to lateral E/e'. We studied the relationships for AASI between right ventricular systolic function, but spearman correlation analysis indicated that AASI was related to TAPSE-S rather than TAPSE (Supplemental table 1).

\section{Logistic regression demonstrating the risk factors of left ventricular diastolic dysfunction}

Univariate regression analysis showed that AASI was associated with increased risk of left ventricular diastolic dysfunction [OR: 2.784, 95\% Confidence interval (CI) 1.137-6.817, P = 0.025] (Data not show). To identity factors that would be independently associated with left ventricular diastolic dysfunction, we 
adjusted for conventional risk factors including age, gender, hypertension, diabetes, coronary artery disease (CAD), chronic obstructive pulmoriary disease (COPD), smoking, drinking, ave-systolic BP and BMI. We found that AASI was still an independent risk factors of left ventricular diastolic dysfunction in patients with HFpEF [OR: 3.261, 95\% Confidence interval (CI) 1.099-9.673, P = 0.033] (Table 3).

\section{ROC curve for AASI to predict left ventricular diastolic dysfunction}

To explore whether AASI provides additional value in predicting impaired left ventricular diastolic function in patients with HFpEF, we conducted ROC curve analysis and found that the AASI had a favorable predictive value for mean $E / e^{\prime}>10$ in patients with HFpEF (AUC $=0.6491, P=0.010$, Fig. 3), with sensitivity and specificity values of $67.8 \%$ and $65.6 \%$, respectively. According to ROC curve analysis, baseline AASI was identified at 0.5248 as the optimal cut-off point to predict left ventricular diastolic dysfunction (Fig. 3).

\section{Discussion}

The major findings of this study were the following: (i) HFpEF with high AASI were more likely to be older, to have higher mean systolic blood pressure and worsen left ventricular diastolic function; (ii) AASI was positively correlated with left ventricular diastolic dysfunction parameters; (iii) AASI might be an independent predictor of left ventricular diastolic dysfunction in patients with HFpEF.

$\mathrm{HFpEF}$ is a group of syndromes with left ventricular diastolic dysfunction as the main clinical manifestation, often accompanied by risk factors such as advanced age, hypertension and diabetes ${ }^{16,17}$, which was consistent with the characteristics of our cohort study. AASI was determined from the records of ABPM has been proposed as a surrogate indicator of arterial stiffness ${ }^{1,2}$. Several studies reported that AASI may be related to diastolic function and prognosis in hypertension and diabetes $3,4,18$. Nevertheless, the role of AASI in patients with HFpEF has not been reported.

In the present study, we found that HFpEF with high AASI were more likely to be older, to have higher systolic blood pressure. This can be explained by the reason that the elderly and high sBP were major factors contributed to arterial stiffness ${ }^{19,20}$. The septal E/e', lateral E/e' and mean $E / e^{\prime}$ are representative parameters that reflect ventricular diastolic dysfunction ${ }^{21}$. As we expected, above mentioned parameters were significant higher in the AASI $>0.55$ group than the AASI $\leq 0.55$ group. Spearman correlation also indicated that AASI was closely positive related to the parameters of diastolic dysfunction. Although E/A value is often regarded as a parameter of diastolic dysfunction, we did not observe the correlation between AASI and E/A in HFpEF. The possible reason was that E/A may have similar values in different stages of diastolic dysfunction ${ }^{22}$. 
Univariate logistic regression analysis found that AASI was associated with increased risk of left ventricular diastolic dysfunction. Previous studies reported that conventional risk factors such as age, hypertension, diabetes, BMI and so on were closely positive related to cardiac dysfunction ${ }^{23,24,25}$. In our study, spearman correlation analysis confirmed that the age and ave-sBP were related to E/e'. These confounding factors may affect the predictive value of AASI in diastolic dysfunction. However, after adjusting for above mentioned risk factors, an independent correlation between AASI and E/e' was still observed in HFpEF. Additionally, we also focused on the right ventricular systolic function, in which the TAPSE and TAPSE-S did not differ between the two groups, indicating that AASI may be a risk factor for left ventricular diastolic dysfunction rather than right ventricular in patients with HFpEF.

Our data showed that the incidence rate of diastolic dysfunction was marked higher in AASI > 0.55 group than AASI $\leq 0.55$ group. ROC curve analysis indicated that the AASI had a favorable predictive value for diastolic dysfunction in patients with HFpEF (sensitivity $67.8 \%$ and specificity $65.6 \%$ ). AASI is one of the major indicators of arterial stiffness. Our finding supported the view that arterial stiffness may serve as risk factors for the development of $\mathrm{HFpEF}^{26,27}$. Severe diastolic dysfunction was associated with an increased risk of major adverse cardiovascular event ${ }^{28}$. Therefore, AASI might be a predictor of adverse events in patients with HFpEF, which needs to be confirmed in the future.

The present study has several limitations that should be considered. First, because this is an observational study, we cannot determine the causality of the results of the study. Second, the sample of this study was small and our findings still need to be further confirmed. Third, because of the small sample, whether AASI was associated with major cardiovascular adverse events in HFpEF could not be determined. Fourth, although we adjusted several risk factors, the effect of potentially unrecognized confounders on the predictive value of AASI in diastolic dysfunction cannot be entirely rule out.

\section{Conclusion}

Increased AASI might be independent associated with left ventricular diastolic dysfunction in patients with HFpEF.

\section{Abbreviations}

AASI: Ambulatory arterial stiffness index; Ave-sBP: Averaged systolic blood pressure; Ave-dBP: Averaged diastolic blood pressure; BMI: Body mass index; CAD: Coronary artery disease; CAVI: Cardio-ankle vascular index; COPD: Chronic obstructive pulmoriary disease; $\mathrm{E}$ : The peak velocity of the filling peak in the early diastolic period; E/A: The E/A ratio; HFpEF: Heart failure with preserved ejection fraction; GM: General measurement; IVSd: Ventricular septal end diastolic thickness; LAVI: Left atrium volume index; LVEF: Left ventricular ejection fraction; LVMI: Left ventricle mass index; LVPWd: Left ventricular posterior wall end diastolic thickness; Lateral E/e': The ratio of early diastolic transmitral flow velocity to mitral annular velocity at the lateral wall; Septal E/e': The ratio of the early diastolic transmitral filling velocity to 
the early diastolic septal tissue velocity; TAPSE: Tricuspid annular plane systolic excursion; TAPSE-S:

Tricuspid annular plane systolic excursion velocity.

\section{Declarations}

\section{Ethics approval and consent to participate}

The study was approved by the institutional ethics board of the First Affiliated Hospital of Chongqing Medical University (approval N0.2020-606) and informed consent was obtained from the patients.

\section{Consent for publication}

Not applicable.

\section{Availability of data and materials}

The data that support the findings of this study are available from the corresponding author upon reasonable request.

\section{Competing interests}

The authors declare that they have no competing interests.

\section{Funding}

The study was supported by National Natural Science Foundation of China, 81970203; National Natural Science Foundation of China, 31800976.

\section{Authors' contributions}

ZQL, XCC, DYZ: study design. DYZ, GLC: financial support. HWZ, WWH, ZQL, LNY: data acquisition. HWZ, YW, JL: data analysis. DJ: technical support. All authors contributed to writing, revising and approved the manuscript.

\section{Acknowledgments}

Not applicable. 


\section{References}

1. Li Y, Wang JG, Dolan E, et al. Ambulatory arterial stiffness index derived from 24-hour ambulatory blood pressure monitoring. Hypertension. 2006;47(3):359-364.

2. Li Y, Dolan E, Wang JG, et al. Ambulatory arterial stiffness index: determinants and outcome. Blood Press Monit. 2006;11(2):107-110.

3. Triantafyllidi $\mathrm{H}$, Tzortzis S, Lekakis J, et al. Association of target organ damage with three arterial stiffness indexes according to blood pressure dipping status in untreated hypertensive patients. Am J Hypertens. 2010;23(12):1265-1272.

4. Dolan E, Thijs L, Li Y, et al. Ambulatory arterial stiffness index as a predictor of cardiovascular mortality in the Dublin Outcome Study. Hypertension. 2006;47(3):365-370.

5. Bastos JM, Bertoquini S, Polónia J. Prognostic significance of ambulatory arterial stiffness index in hypertensives followed for 8.2 years: its relation with new events and cardiovascular risk estimation. Rev Port Cardiol. 2010;29(9):1287-1303.

6. Zile MR, Gottdiener JS, Hetzel SJ, et al. Prevalence and significance of alterations in cardiac structure and function in patients with heart failure and a preserved ejection fraction. Circulation. 2011;124(23):2491-2501.

7. Shah SJ, Kitzman DW, Borlaug BA, et al. Phenotype-Specific Treatment of Heart Failure With Preserved Ejection Fraction: A Multiorgan Roadmap. Circulation. 2016;134(1):73-90.

8. van Riet EE, Hoes AW, Wagenaar KP, Limburg A, Landman MA, Rutten FH. Epidemiology of heart failure: the prevalence of heart failure and ventricular dysfunction in older adults over time. A systematic review. Eur J Heart Fail. 2016;18(3):242-252.

9. Zern EK, Ho JE, Panah LG, et al. Exercise Intolerance in Heart Failure With Preserved Ejection Fraction: Arterial Stiffness and Aabnormal Left Ventricular Hemodynamic Responses During Exercise. J Card Fail. 2021;27(6):625-634.

10. Chirinos JA, Bhattacharya P, Kumar A, et al. Impact of Diabetes Mellitus on Ventricular Structure, Arterial Stiffness, and Pulsatile Hemodynamics in Heart Failure With Preserved Ejection Fraction. J Am Heart Assoc. 2019;8(4):e011457.

11. Cuijpers I, Simmonds SJ, van Bilsen M, et al. Microvascular and lymphatic dysfunction in HFpEF and its associated comorbidities. Basic Res Cardiol. 2020;115(4):39. Published 2020 May 25.

12. Takagi $\mathrm{K}$, Ishihara $\mathrm{S}$, Kenji $\mathrm{N}$, et al. Clinical significance of arterial stiffness as a factor for hospitalization of heart failure with preserved left ventricular ejection fraction: a retrospective matched case-control study. J Cardiol. 2020;76(2):171-176.

13. Pieske B, Tschöpe $C$, de Boer RA, et al. How to diagnose heart failure with preserved ejection fraction: the HFA-PEFF diagnostic algorithm: a consensus recommendation from the Heart Failure Association (HFA) of the European Society of Cardiology (ESC) [published correction appears in Eur Heart J. 2021 Mar 31;42(13):1274]. Eur Heart J. 2019;40(40):3297-3317. 
14. Adiyaman A, Dechering DG, Boggia J, et al. Determinants of the ambulatory arterial stiffness index in 7604 subjects from 6 populations [published correction appears in Hypertension. 2009 Feb;53(2):e20]. Hypertension. 2008;52(6):1038-1044.

15. Hu R, Mazer CD, Tousignant C. Relationship between tricuspid annular excursion and velocity in cardiac surgical patients. J Cardiothorac Vasc Anesth. 2014;28(5):1198-1202.

16. Pagel PS, Tawil JN, Boettcher BT, et al. Heart Failure With Preserved Ejection Fraction: A Comprehensive Review and Update of Diagnosis, Pathophysiology, Treatment, and Perioperative Implications. J Cardiothorac Vasc Anesth. 2021;35(6):1839-1859.

17. Myhre PL, Selvaraj S, Solomon SD. Management of hypertension in heart failure with preserved ejection fraction: is there a blood pressure goal?. Curr Opin Cardiol. 2021;36(4):413-419.

18. Palmas W, Pickering T, Eimicke JP, et al. Value of ambulatory arterial stiffness index and 24-h pulse pressure to predict progression of albuminuria in elderly people with diabetes mellitus. Am J Hypertens. 2007;20(5):493-500.

19. Sun Z. Aging, arterial stiffness, and hypertension. Hypertension. 2015;65(2):252-256.

20. Boutouyrie P, Chowienczyk P, Humphrey JD, Mitchell GF. Arterial Stiffness and Cardiovascular Risk in Hypertension. Circ Res. 2021;128(7):864-886.

21. Nauta JF, Hummel YM, van der Meer P, Lam CSP, Voors AA, van Melle JP. Correlation with invasive left ventricular filling pressures and prognostic relevance of the echocardiographic diastolic parameters used in the 2016 ESC heart failure guidelines and in the 2016 ASE/EACVI recommendations: a systematic review in patients with heart failure with preserved ejection fraction. Eur J Heart Fail. 2018;20(9):1303-1311.

22. Mitter SS, Shah SJ, Thomas JD. A Test in Context: E/A and E/e' to Assess Diastolic Dysfunction and LV Filling Pressure. J Am Coll Cardiol. 2017;69(11):1451-1464.

23. Sundqvist MG, Sahlén A, Ding ZP, Ugander M. Diastolic function and its association with diabetes, hypertension and age in an outpatient population with normal stress echocardiography findings. Cardiovasc Ultrasound. 2020;18(1):46. Published 2020 Nov 20.

24. Yan WF, Gao Y, Zhang Y, et al. Impact of type 2 diabetes mellitus on left ventricular diastolic function in patients with essential hypertension: evaluation by volume-time curve of cardiac magnetic resonance. Cardiovasc Diabetol. 2021;20(1):73. Published 2021 Mar 25.

25. Alpert MA, Omran J, Mehra A, Ardhanari S. Impact of obesity and weight loss on cardiac performance and morphology in adults. Prog Cardiovasc Dis. 2014;56(4):391-400.

26. Pandey A, Khan H, Newman AB, et al. Arterial Stiffness and Risk of Overall Heart Failure, Heart Failure With Preserved Ejection Fraction, and Heart Failure With Reduced Ejection Fraction: The Health ABC Study (Health, Aging, and Body Composition). Hypertension. 2017;69(2):267-274.

27. Chi C, Liu Y, Xu Y, Xu D. Association Between Arterial Stiffness and Heart Failure With Preserved Ejection Fraction. Front Cardiovasc Med. 2021;8:707162.

28. Kane GC, Karon BL, Mahoney DW, et al. Progression of left ventricular diastolic dysfunction and risk of heart failure. JAMA. 2011;306(8):856-863. 


\section{Tables}

Table 1

Baseline clinical characteristics of included patients with HFpEF. 


\begin{tabular}{|c|c|c|c|c|}
\hline Characteristics & $\begin{array}{l}\text { All } \\
(\mathrm{N}=91)\end{array}$ & $\begin{array}{l}A A S I \leq 0.55 \\
(N=45)\end{array}$ & $\begin{array}{l}\text { AASI>0.55 } \\
(\mathrm{N}=46)\end{array}$ & $P$ \\
\hline Age(years) & $68.73 \pm 13.93$ & $63.78 \pm 14.64$ & $73.57 \pm 11.41$ & $<0.001$ \\
\hline Female & $54(59 \%)$ & $26(58 \%)$ & $28(61 \%)$ & 0.764 \\
\hline \multicolumn{5}{|l|}{ History } \\
\hline Smoking & $14(15 \%)$ & $5(11 \%)$ & $9(20 \%)$ & 0.264 \\
\hline Drinking & $23(25 \%)$ & $11(24 \%)$ & $12(26 \%)$ & 0.857 \\
\hline Hypertension & $60(66 \%)$ & $26(58 \%)$ & $34(74 \%)$ & 0.104 \\
\hline CAD & $41(45 \%)$ & $18(40 \%)$ & $23(50 \%)$ & 0.338 \\
\hline Diabetes & $30(33 \%)$ & $12(27 \%)$ & 18(39\%) & 0.206 \\
\hline COPD & $10(11 \%)$ & $6(13 \%)$ & $4(9 \%)$ & 0.479 \\
\hline \multicolumn{5}{|l|}{ GM } \\
\hline Height(m) & $1.59 \pm 0.08$ & $1.59 \pm 0.08$ & $1.59 \pm 0.08$ & 0.905 \\
\hline Weight(kg) & $59.73 \pm 9.88$ & $59.64 \pm 10.29$ & $59.82 \pm 9.58$ & 0.931 \\
\hline BMI & $23.60 \pm 3.35$ & $23.62 \pm 3.76$ & $23.57 \pm 2.92$ & 0.941 \\
\hline Ave-sBP(mmHg) & $121.20 \pm 17.04$ & $116.29 \pm 15.10$ & $126.00 \pm 17.60$ & 0.006 \\
\hline Ave-dBP(mmHg) & $68.77 \pm 8.24$ & $69.40 \pm 7.10$ & $68.15 \pm 9.26$ & 0.473 \\
\hline AASI & $0.54 \pm 0.19$ & $0.39 \pm 0.14$ & $0.69 \pm 0.10$ & $<0.001$ \\
\hline \multicolumn{5}{|l|}{ Echo data } \\
\hline IVSd(mm) & $11.08 \pm 1.87$ & $10.93 \pm 1.77$ & $11.22 \pm 1.98$ & 0.468 \\
\hline LVPWd(mm) & $10.55 \pm 1.44$ & $10.52 \pm 1.50$ & $10.58 \pm 1.39$ & 0.858 \\
\hline $\operatorname{LAVI}\left(\mathrm{ml} / \mathrm{m}^{2}\right)$ & $35.32 \pm 13.73$ & $32.21 \pm 12.51$ & $38.15 \pm 14.30$ & 0.047 \\
\hline $\operatorname{LVMI}\left(\mathrm{g} / \mathrm{m}^{2}\right)$ & $118.62 \pm 37.68$ & $114.86 \pm 34.03$ & $122.20 \pm 40.92$ & 0.364 \\
\hline LVEF(\%) & $62.29 \pm 5.453$ & $62.84 \pm 5.94$ & $61.74 \pm 4.94$ & 0.337 \\
\hline$E$ & $69.62 \pm 23.55$ & $64.02 \pm 20.05$ & $75.10 \pm 25.58$ & 0.024 \\
\hline$E / A$ & $0.82 \pm 0.36$ & $0.83 \pm 0.36$ & $0.82 \pm 0.36$ & 0.865 \\
\hline Mean E/e' & $12.68 \pm 4.73$ & $11.34 \pm 4.22$ & $13.99 \pm 4.88$ & 0.007 \\
\hline Septal E/e' & $14.63 \pm 5.90$ & $13.29 \pm 5.89$ & $15.95 \pm 5.67$ & 0.031 \\
\hline Lateral E/e' & $10.73 \pm 4.25$ & $9.39 \pm 3.26$ & $12.04 \pm 4.72$ & 0.003 \\
\hline
\end{tabular}




$\begin{array}{llrrr}\text { TAPSE } & 19.17 \pm 3.91 & 18.73 \pm 4.15 & 19.59 \pm 3.66 & 0.302 \\ \text { TAPSE-S } & 11.73 \pm 2.70 & 11.62 \pm 3.10 & 11.84 \pm 2.29 & 0.704\end{array}$

AASI: Ambulatory arterial stiffness index, Ave-sBP: Averaged systolic blood pressure, Ave-dBP: Averaged diastolic blood pressure, BMI: Body mass index, CAD: Coronary artery disease, COPD: chronic obstructive pulmoriary disease, $\mathrm{E}$ : the peak velocity of the filling peak in the early diastolic period, E/A: the E/A ratio, GM: General measurement, IVSd: Ventricular septal end diastolic thickness, LAVI: left atrium volume index, LVEF: left ventricular ejection fraction, LVMI: left ventricle mass index, LVPWd: Left ventricular posterior wall end diastolic thickness, Lateral E/e': the ratio of early diastolic transmitral flow velocity to mitral annular velocity at the lateral wall, Septal E/e': the ratio of the early diastolic transmitral filling velocity to the early diastolic septal tissue velocity, TAPSE: tricuspid annular plane systolic excursion, TAPSE-S: tricuspid annular plane systolic excursion velocity.

Table 2

Spearman correlations between clinical characteristics and left ventricular diastolic function

\begin{tabular}{|lllllllll|}
\hline & \multicolumn{7}{l}{ Mean E/e' } & \multicolumn{2}{l}{ Septal E/e' } & \multicolumn{2}{l}{ Lateral E/e' } & \\
\hline & $r$ & $P$ & $r$ & $P$ & $r$ & $P$ & $r$ & $P$ \\
\hline Age & 0.285 & 0.006 & 0.270 & 0.010 & 0.274 & 0.009 & 0.192 & 0.068 \\
\hline Height & -0.108 & 0.306 & -0.111 & 0.296 & -0.123 & 0.245 & -0.094 & 0.252 \\
\hline BMI & 0.042 & 0.690 & -0.024 & 0.823 & 0.144 & 0.174 & -0.085 & 0.424 \\
\hline Ave-sBP & 0.316 & 0.002 & 0.280 & 0.007 & 0.330 & 0.001 & 0.131 & 0.214 \\
\hline Ave-dBP & 0.036 & 0.734 & -0.014 & 0.895 & 0.109 & 0.303 & -0.096 & 0.366 \\
\hline AASI & 0.290 & 0.005 & 0.237 & 0.024 & 0.295 & 0.004 & 0.262 & 0.012 \\
\hline
\end{tabular}

Table 3

Multivariate logistic regression demonstrating the risk factors of diastolic dysfunction. 


\begin{tabular}{|c|c|c|c|}
\hline \multirow[b]{2}{*}{ Risk factors } & \multicolumn{3}{|c|}{ Mean $E / e^{\prime}>10$} \\
\hline & OR & $95 \% \mathrm{Cl}$ & $P$ \\
\hline AASI>0.55 & 3.261 & $1.099-9.673$ & 0.033 \\
\hline Age $>65$ & 1.046 & $0.292-3.745$ & 0.945 \\
\hline Female & 1.429 & $0.355-5.746$ & 0.615 \\
\hline Hypertension & 1.137 & $0.292-4.427$ & 0.853 \\
\hline Diabetes & 1.278 & $0.413-3.951$ & 0.671 \\
\hline CAD & 1.807 & $0.595-5.490$ & 0.296 \\
\hline COPD & 4.905 & $0.686-35.084$ & 0.113 \\
\hline Smoking & 0.969 & $0.207-4.542$ & 0.968 \\
\hline Drinking & 0.316 & $0.075-1.336$ & 0.117 \\
\hline Ave-sBP > 135 & 1.552 & $0.348-6.931$ & 0.565 \\
\hline $\mathrm{BMI}>25$ & 2.018 & $0.670-6.077$ & 0.212 \\
\hline
\end{tabular}

\section{Figures}




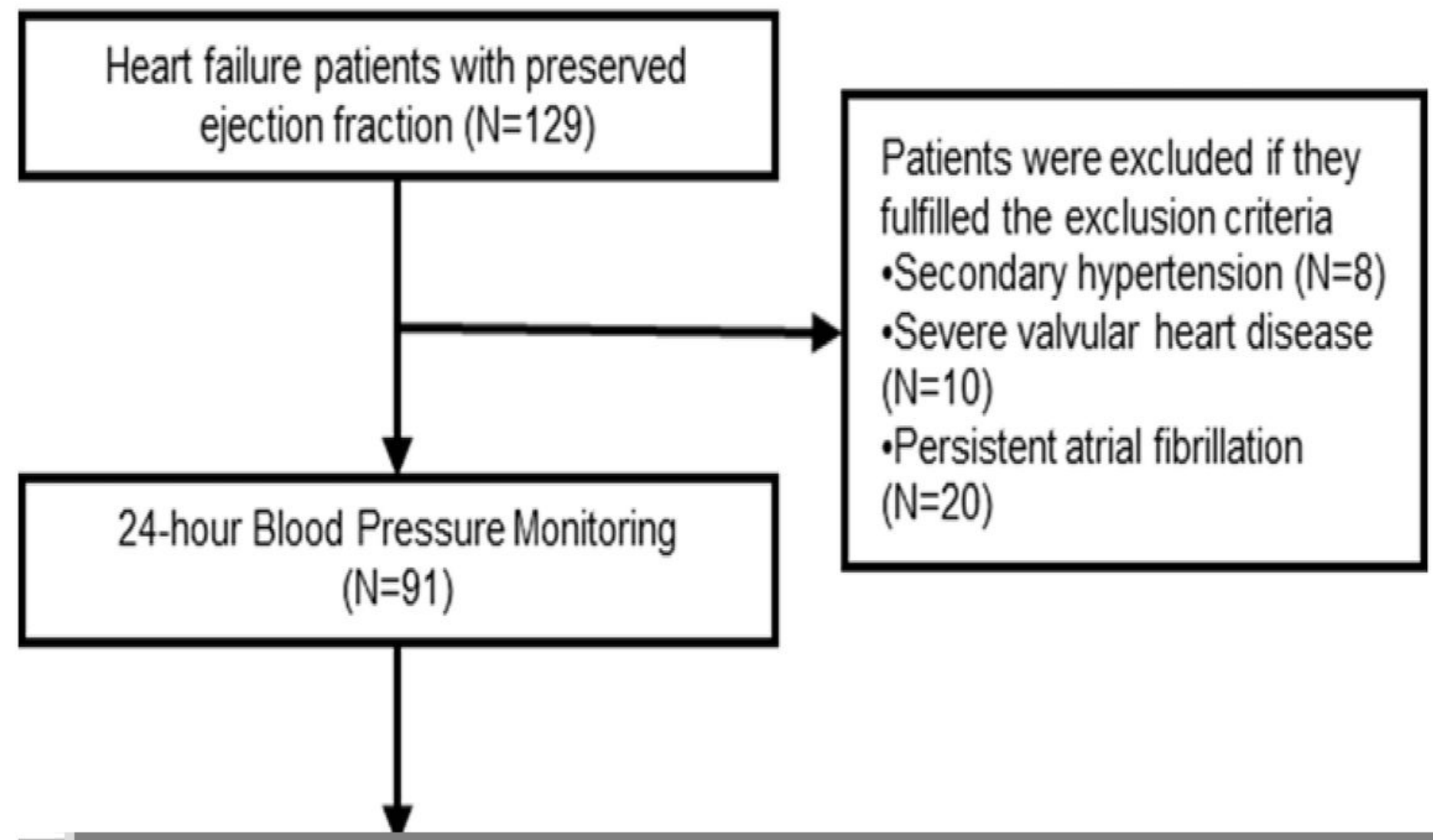

Figure 1

Flow diagram for the study population.

Figure 2

Correlation of the AASI with left ventricular diastolic function. 
Figure 3

ROC curves of AASI for left ventricular diastolic dysfunction.

\section{Supplementary Files}

This is a list of supplementary files associated with this preprint. Click to download.

- SDC.docx 